\title{
Las dimensiones espaciales del manejo de crisis. Interés de la investigación y aplicación en Quito
}

Les dimensions spatiales de la gestion des crises. Intérêt de la recherche et application à Quito

Spatial dimensions of crisis management. Relevance of the research and application in Quito

Robert D'Ercole y Pascale Metzger

\section{OpenEdition}

Journals

Edición electrónica

URL: http://journals.openedition.org/bifea/2543

DOI: 10.4000/bifea.2543

ISSN: 2076-5827

Editor

Institut Français d'Études Andines

Edición impresa

Fecha de publicación: 1 diciembre 2009

Paginación: 893-915

ISSN: 0303-7495

Referencia electrónica

Robert D'Ercole y Pascale Metzger, «Las dimensiones espaciales del manejo de crisis. Interés de la investigación y aplicación en Quito », Bulletin de l'Institut français d'études andines [En línea], 38 (3) | 2009, Publicado el 01 junio 2010, consultado el 17 noviembre 2020. URL : http:// journals.openedition.org/bifea/2543; DOI : https://doi.org/10.4000/bifea.2543 


\title{
Las dimensiones espaciales del manejo de crisis. Interés de la investigación y aplicación en Quito
}

\author{
Robert D'Ercole* \\ Pascale Metzger*
}

\begin{abstract}
Resumen
Considerando los límites de la acción preventiva y las debilidades de los sistemas de manejo de crisis, el artículo busca demostrar el interés de un enfoque espacial y la necesidad de desarrollar investigaciones sobre este tema. Se trata de reflexionar sobre los tipos de espacios y de territorios involucrados en el manejo de crisis que hay que tomar en cuenta en estas investigaciones. Se propone un ejemplo de aplicación con el caso de Quito. Se trata de identificar los espacios de la gestión de crisis dentro del territorio metropolitano y relacionar los espacios en los que la población es particularmente vulnerable con los espacios recursos en materia de oferta de servicios médicos. El ejemplo muestra que el enfoque espacial del manejo de crisis permite abrir el estrecho marco de la preparación para los desastres, estableciendo vínculos entre preparación, prevención y planificación preventiva.
\end{abstract}

Palabras clave: manejo de crisis, enfoque espacial, vulnerabilidad, preparación, prevención, planificación preventiva, Quito

* Institut de Recherche pour le Développement (IRD), UR 029, programa Pacivur. Calle Teruel 357, Miraflores, Casilla 18-1209, Lima 18, Perú. E-mails:

robert.dercole@ird.fr

pascale.metzger@ird.fr 


\title{
Les dimensions spatiales de la gestion des crises. Intérêt de la recherche et application à Quito
}

\begin{abstract}
Résumé
Compte tenu des limites de l'action préventive et des faiblesses des systèmes de gestion des crises, l'article vise à démontrer l'intérêt de l'approche spatiale de la gestion des crises et la nécessité de développer des recherches sur cette question. Une réflexion est menée sur les types d'espaces et de territoires de la gestion des crises à prendre en considération dans ces recherches. Un exemple d'application est proposé avec le cas de Quito en identifiant les espaces de la gestion des crises au sein du territoire métropolitain et en mettant en relation les espaces où la population est particulièrement vulnérable avec les espaces ressources en matière d'offre de soins médicaux. L'exemple montre que l'approche spatiale de la gestion des crises permet de sortir du cadre étroit de la préparation aux catastrophes en établissant des liens entre préparation, prévention et planification préventive.
\end{abstract}

Mots clés: gestion des crises, approche spatiale, vulnérabilité, préparation, prévention, planification préventive, Quito

\section{Spatial dimensions of crisis management. Relevance of the research and application in Quito}

\begin{abstract}
Considering the limits of preventive action and the weaknesses in the systems of crisis management, this article aims to demonstrate the relevance of a spatial approach to crisis management and the need to enlarge research on this issue. It is necessary to reflect upon the types of spaces and the territories involved in the management of crises being treated in these investigations. The case of Quito is proposed as an example of the application of this approach. We identify crisis management areas in the Quito metropolitan district in order to link areas where the population is particularly vulnerable with areas of health care resources. The example shows that the spatial approach of crisis management permit to go beyond disaster preparedness, establishing relationships between preparedness, prevention and mitigation planning.
\end{abstract}

Key words: crisis management, spatial approach, vulnerability, preparedness, prevention, preventive planning, Quito

Las crisis en medio urbano corresponden a «situaciones de desarreglo organizacional y de desórdenes en cadena que afectan a lo social, lo económico y al ambiente» (Dubois-Maury \& Chaline, 2002: 175). Están vinculadas a eventos mayores que pueden ser causados por fenómenos destructores de origen natural o antrópico y que provocan la superación de las «capacidades de autorregulación interna que de ordinario garantizan la continuidad del funcionamiento urbano» (Dubois-Maury \& Chaline, 2002: 175). Son rupturas o muy fuertes perturbaciones, en dimensiones materiales y logísticas, pero también económicas, sociales o sanitarias, que dibujan los contornos de las crisis que afectan a las sociedades y territorios. Pueden ser provocadas por un sismo o una epidemia, una inundación o un movimiento social, un deslizamiento de terreno o una situación política. 
En un pasado todavía reciente, el manejo de las crisis era considerado como una preocupación dictada por la fuerza de los eventos, un último recurso por ausencia de prevención. Este manejo ocupa progresivamente un lugar importante en la gestión de los riesgos. El número cada vez más importante de investigadores que le prestan interés muestra que la crisis como objeto de investigación en el campo del riesgo ha crecido, cuando esta problemática era, hace algunos años, un asunto principalmente reservado a los especialistas de protección civil. Este interés reciente de los investigadores por el tema de las crisis ofrece la posibilidad de desenmarañar su complejidad, de reducir las incertidumbres y, a fin de cuentas, de disminuir la vulnerabilidad de los territorios y de su población.

Las situaciones de gran inestabilidad que constituyen las crisis pueden delimitarse en el tiempo, con un «antes», un «durante» y un «después»1, es decir un periodo fuera de la crisis, un periodo de crisis y un periodo poscrisis ${ }^{2}$. El periodo de crisis propiamente dicho presenta dos o tres fases sucesivas según existan o no fenómenos anunciadores:

- Una fase de pre emergencia marcada por la existencia de fenómenos precursores (actividad sísmica anormal registrada en un volcán, averías recurrentes en una fábrica que almacena o procesa productos peligrosos, amenazas de levantamiento social, etc.), que ponen en alerta a las autoridades y a los servicios involucrados, incluso a la población mediante una información preventiva.

- Una fase de emergencia, marcada por la implementación de los auxilios y la protección de las personas y de los bienes, que se inicia en el momento en que la amenaza es inminente o cuando se produce el impacto si se trata de un fenómeno imprevisible. Esta fase, que puede durar de algunas horas a varios días, cubre todo el periodo del impacto y no termina hasta que se hayan tomado todas las medidas de auxilio y de protección.

- Una fase de recuperación (igualmente llamada fase de retorno a la autonomía o fase de normalización inmediata) que puede durar de algunos días a varios meses, en los casos más graves. Se prolonga hasta el restablecimiento de las condiciones mínimas indispensables para un retorno a una situación aceptable (aún cuando sea diferente a la situación inicial) en los sectores afectados3.

Más allá de este enfoque de la crisis mediante fases temporales, este artículo busca mostrar la pertinencia científica de una investigación geográfica sobre su manejo. La producción de conocimientos sobre las dimensiones espaciales de la gestión de crisis se justifica a la vez por las limitaciones constatadas de la acción preventiva y

1 Varios autores siguen este tipo de corte como Denis (1993), Dirección General de Protección Civil Española (1997), Lutoff (2000).

2 Periodo de reconstrucción, igualmente llamado periodo de normalización a largo plazo.

3 Durante esta fase se efectúan las primeras operaciones de rehabilitación, en particular: control del estado de las construcciones; refuerzo o destrucción de las construcciones dañadas, según su estado; limpieza de las viviendas y de las vías de comunicación; reparación de los daños más importantes sufridos por las infraestructuras de transporte, de telecomunicaciones y de abastecimiento en agua; reparación de la red eléctrica y de aprovisionamiento en combustibles; reubicación temporal de las personas sin techo, etc. (Dirección General de Protección Civil Española, 1997). 
por el desconocimiento de las dificultades espaciales, propias del manejo de crisis. En una primera etapa, este artículo retrata la evolución de la investigación en el campo de la crisis, la insuficiencia de las investigaciones espacializadas y el interés de los conocimientos que pueden aportar. En una segunda etapa, la reflexión atañe a los tipos de espacios y territorios del manejo de las crisis. El análisis de sus articulaciones permite dar cuenta de la dimensión espacial de la gestión de crisis y evidenciar algunas vulnerabilidades de los sistemas de manejo. La última parte es un primer ejemplo de aplicación de este enfoque en el caso de Quito, donde se identifican los espacios del manejo de crisis dentro del territorio metropolitano y se relacionan los espacios en los que la población es particularmente vulnerable con los espacios recursos en materia de oferta de servicios médicos.

\section{EL MANEJO DE CRISIS EN MEDIO URBANO: UN TEMA QUE HA PASADO A SER ESENCIAL}

\section{1. Los límites de la prevención}

El conocimiento de los procesos físicos y humanos que dan lugar a desastres de origen natural ha incrementado claramente durante estas últimas décadas, con el mejoramiento de los medios técnicos que permiten preverlos e intervenir de manera preventiva. Sin embargo, se observa paralelamente un crecimiento muy sensible de las víctimas y de las pérdidas materiales y financieras provocadas por los desastres. Así la lista de las ciudades afectadas por un desastre mayor no ha cesado de alargarse y de diversificarse (Dubois-Maury \& Chaline, 2002), sobre todo en los países del sur.

Esta paradoja tiene múltiples causas (D’Ercole, 1994; Pigeon, 1996; Pelling, 2003): desarrollo de las superficies urbanizadas en zonas de riesgo, sistemas inapropiados de construcción, incremento de la población urbana pobre y no preparada, ausencia de medios financieros para la reducción de las vulnerabilidades, complejidad creciente de los organismos urbanos, debilidad institucional de la planificación urbana y de la legislación, etc. La paradoja es, al mismo tiempo, reveladora de las distorsiones observables entre las políticas de gestión de los riesgos y los riesgos evaluados por los científicos. En otros términos, si la producción científica en materia de riesgo puede considerarse abundante (aunque desigual en cuanto a los objetos de estudio y en el plano cualitativo), se basa en un enfoque conceptual inadecuado (D’Ercole \& Metzger, 2009) y desemboca en conocimientos inaplicables o en recomendaciones escasamente tomadas en cuenta por los tomadores de decisión.

En la mayoría de las ciudades de los países en desarrollo, los espacios de riesgos ya están urbanizados (Sierra, 2000) y el proceso de densificación de estos espacios prosigue ineluctablemente. 
Al mismo tiempo, las obras de protección, aunque útiles en algunos casos, han mostrado límites considerables e incluso acrecientan a veces los riesgos. Así, varios autores, como Blaikie et al. (1994), Saury-Pujol et al. (2001), Tunstall (2004), Pigeon (2006) o Werritty (2006) reconocen la escasa eficiencia de las políticas preventivas de gestión del riesgo (planificación territorial o protección tecnológica) y concuerdan en la imposibilidad de eliminar los riesgos. La cuestión planteada por la optimización del manejo de las crisis se vuelve entonces esencial, en particular en los países del sur dentro de los cuales gran número de territorios están expuestos a riesgos de origen natural.

\section{2. Las debilidades de los sistemas de manejo de crisis}

La necesidad de escudriñar el manejo de las crisis se debe no solo a los límites de la acción preventiva, sino igualmente al carácter inoperante de los sistemas de manejo de crisis existentes, sobre todo en los países en desarrollo. Numerosas son las investigaciones que van en este sentido. Para no mencionar sino ejemplos latinoamericanos, citemos a Voight (1990) sobre la erupción del Nevado del Ruiz en Colombia y la desaparición de la ciudad de Armero en 1985; Rocha \& Christoplos (2001) sobre el huracán Mitch en Nicaragua en 1998; Lane et al. (2003) sobre la erupción del volcán Tungurahua y la difícil evacuación de la ciudad de Baños en el Ecuador en 1999; D’Ercole et al. (2007) a propósito del sismo del 15 de agosto de 2007 en Pisco (Perú). Todos estos análisis evidencian las dificultades inherentes a la gestión de estas situaciones complejas y marcadas por la incertidumbre. Los problemas destacados se refieren más a la debilidad de las instituciones, en materia de organización y de coordinación, y a los problemas de comunicación que a los aspectos económicos y materiales. La falta de anticipación, la ausencia de preparación, el desconocimiento de los lugares afectados y de sus capacidades de reacción, la carencia de articulación entre el nivel nacional y local, entre las autoridades y la población, la ignorancia de los procesos sociales y espaciales pero también políticos e instituciones involucrados en la crisis terminan generalmente en gestión de emergencia improvisada.

El análisis de Patrick Lagadec sobre la crisis «fuera de las normas», generada por el huracán Katrina en la Nueva Orleans en 2005, aunque se refiere a los Estados Unidos, podría aplicarse perfectamente a los países en desarrollo. El «fiasco Katrina» está ligado sobre todo a

«la incapacidad del sistema para aportar en tiempo y hora la información requerida a los lugares requeridos y a la falta de imaginación, de iniciativa, de liderazgo, de coordinación, de anticipación, de vigilancia, de flexibilidad, de agilidad, de preparación» (Lagadec, 2007).

En los países en desarrollo, se ha podido observar crisis de escasa magnitud que dan origen a disfunciones mayores. Es el caso de Quito, en 1999, durante la caída de ceniza producida por la erupción del volcán Pichincha (D’Ercole \& Metzger, 2000) o de La Paz durante las inundaciones del 19 de febrero de 2002 (O'Hare \& Rivas, 2005) —ver también el artículo de R. D’Ercole, P. Metzger y A. Sierra en este volumen, pp. 487-499-. 


\section{3. Las investigaciones sobre la crisis}

Es impresionante el volumen de investigación producido, desde hace medio siglo, sobre el tema de los riesgos de origen natural. Esta investigación, dedicada en lo esencial al conocimiento de las amenazas, comenzó a diversificarse en 1980 y sobre todo en la década de 1990, bajo el impulso del Decenio Internacional para la Reducción de los Desastres Naturales instituido por las Naciones Unidas. La visión de los riesgos es hoy en día más global, incluye los elementos esenciales, la vulnerabilidad, y, más recientemente, la resiliencia y la adaptabilidad, temas que se encuentran frecuentemente asociados a los estudios que atañen al cambio climático, o también a la «transformabilidad» de los sistemas socioecológicos (Walker et al., 2004).

Por su parte, la investigación sobre la crisis y su manejo ha progresado sensiblemente durante los últimos 40 años, tras los estudios precursores (esencialmente anglosajones), entre los cuales descuellan Quarantelli, Dynes, Drabeck, Perry, Lindell, Greene, Leik, Hultaker, etc.4. En los años 1970-1980, varios estudios en sociología de los desastres han analizado catástrofes naturales y accidentes tecnológicos, en un contexto ante todo norteamericano. Los estudios de los años 1980-1990 se enfocaron más sobre las definiciones, tipologías y tiempos de la crisis, las exigencias para la acción y el principio de precaución (Lagadec, 2003). Frente a los innumerables estudios realizados en el campo de los riesgos, Patrick Lagadec lamenta las debilidades de la reflexión emprendida sobre el manejo de las crisis, precisamente cuando el cambio de dimensión de las crisis actuales (mayor complejidad, mayor incertidumbres) necesitaría más estudios en el tema. Sin embargo, Lagadec destaca el esfuerzo creciente que se ha hecho en materia de investigación sobre la crisis en estos últimos 15 años en Europa, y en particular en Francia, gracias al sociólogo Claude Gilbert6. Desde el punto de vista de las disciplinas científicas, la impulsión ha venido de la sociología, de la psicología, de la antropología, de las ciencias políticas y de la comunicación (Lagadec, 2003; Gilbert, 2005). Otros especialistas también están involucrados como médicos, economistas e ingenieros de la corriente de la cindínica (Kerven \& Wybo, 2002).

Las investigaciones sobre el manejo de crisis se han interesado primero en el análisis de las crisis ligadas a accidentes y desastres, poniendo énfasis sobre su naturaleza, su magnitud y sobre las reacciones específicas de las personas, grupos y organizaciones (Alexander, 2000; Veyret et al., 2004). Después se centraron en las relaciones entre crisis, incertidumbre y precaución, en el surgimiento de situaciones desconocidas, que superan los marcos de referencia habituales

4 Ver en particular la bibliografía de la obra de Quarantelli (1998).

5 En este orden de ideas, ver en particular en este volumen el artículo de J. Robert, R. D’Ercole, P. Pigeon y T. Serrano (pp. 709-733) a propósito de complejidad y de incertidumbre frente a una posible erupción volcánica y el de P. Metzger y R. D’Ercole (pp. 917-936) sobre la transmisión de vulnerabilidad.

6 Ver los estudios del GIS «riesgos colectivos y situaciones de crisis» < http//www.msh-alpes.prd.fr/ gisrisques/>. 
(Godard et al., 2002). Muy a menudo, las disfunciones humanas y técnicas en la gestión de las crisis, evidenciadas por las investigaciones y análisis de experiencias, constatan fallas en la apreciación de los fenómenos espaciales, de las dinámicas o interacciones espaciales y subrayan la ausencia de preparación en estos campos. Como lo sugiere Lagadec (2007), en el manejo de crisis, lo que está en juego son las texturas profundas de las sociedades, así como las redes y las interdependencias que el análisis espacial contribuye a aprehender. Las situaciones de crisis proyectan a los actores dentro de un universo en el que las referencias habituales son súbitamente inapropiadas. Estas últimas están inscritas en los modos de pensar y de actuar, pero también se trata de referencias espaciales. En efecto, el manejo de una crisis es el manejo de movimientos rápidos y desordenados en el seno de un territorio en el cual los espacios y los flujos ya no son los del funcionamiento territorial normal. Por consiguiente, los enfoques espaciales y territoriales son necesarios y complementarios de los demás enfoques científicos del manejo de las crisis. Ahora bien, se trata de campos de investigación poco explorados por los geógrafos, cuyo aporte a esta problemática permanece muy discreto.

\section{4. Los geógrafos y el enfoque espacial de la crisis}

Un balance reciente sobre la investigación geográfica en materia de riesgo (Morel et al., 2006) destaca la importancia de los estudios sobre las amenazas en los años 1970-1980, seguida de un numero creciente de investigaciones sobre la interfaz hombre/naturaleza, sobre los diferentes factores de vulnerabilidad de las sociedades, antes de abordar enfoques más globales y territoriales. La investigación se hace más pluridisciplinaria desde fines de la década de 1990, los geógrafos asociándose cada vez más a otros especialistas (sociólogos, economistas, etc.). Sin embargo, el balance de estos estudios revela una implicancia insuficiente de la geografía en el campo del manejo de las crisis. Esta disciplina ha desarrollado principalmente unos análisis de los efectos sociales y territoriales de los desastres, y de los procesos de rehabilitación y reconstrucción poscrisis. Son investigaciones realizadas tras desastres de gran magnitud: la erupción del volcán Pinatubo en Filipinas (Gaillard, 2001), el tsunami asiático de diciembre de 2004 (Ozier \& De Longueville, 2005; Lavigne \& Paris, 2006); el huracán Katrina en Nueva Orleans (Mancebo, 2006; Curtis et al., 2007); o tras otras situaciones generadas por riesgos manifiestos o potenciales de menor magnitud (D’Ercole, 1996; Bimal Kanti, 2005; Gaillard et al., 2007; D’Ercole et al., 2007; Lane et al., 2003).

Generalmente el tema de la crisis es abordado de manera indirecta o parcial. Así, los estudios de algunos geógrafos abordan el tema del manejo de crisis dentro del marco global de la vulnerabilidad (Chardon, 1996; Cutter et al., 2000; Weichselgartner, 2001). Estas investigaciones ponen énfasis en las condiciones físicas y socioeconómicas que pueden llevar a crisis y desarrollan enfoques espaciales y territoriales de sumo interés. Pero estos estudios no se focalizan en el manejo de la crisis en sí. Ocurre lo mismo con el desarrollo de herramientas para el diagnóstico de vulnerabilidades adaptadas a las necesidades de los actores locales, 
en las cuales la vulnerabilidad ligada a las modalidades del manejo de la crisis no constituye sino una de las dimensiones de las vulnerabilidades consideradas (Meur-Ferrec et al., 2004; Pottier, 2006). Manteniendo al mismo tiempo una lógica de vulnerabilidad social y territorial, algunas otras investigaciones geográficas han penetrado directamente el campo de la crisis. Se trata en particular de estudios relativos a los comportamientos humanos en periodo de emergencia, de cartografía de los lugares de seguridad, percibidos por la poblacion expuesta al riesgo volcánico (D'Ercole, 1991) o de reflexiones sobre los elementos esenciales de un territorio para el manejo de crisis (D’Ercole \& Metzger, 2004; D’Ercole et al., 2005).

Algunos estudios de geógrafos han abordado las dimensiones espaciales y territoriales del manejo de las crisis desde ángulos particulares. Es el caso de la problemática de la movilidad y de la accesibilidad que permite prever y anticipar las consecuencias de fallas técnicas, de aislamiento de territorios, de parálisis urbanas en tiempo de crisis (Demoraes, 2004; Voiron-Canicio \& Olivier, 2005; Appert \& Chapleton, 2008), o de transporte de materias peligrosas (Griot, 2007). Los comportamientos de muchedumbre en medio urbano y en periodo de crisis también han sido objeto de reflexiones (Provitolo, 2005), así como los comportamientos de algunos actores clave de la crisis (D’Ercole \& Moncayo, 1991) que evidencian las dificultades institucionales previsibles (Serrano, 2007). Las representaciones de los riesgos y las diferencias de puntos de vista entre población expuesta, científicos y otros actores, sobre todo en materia de espacios amenazados, pueden influenciar los comportamientos en tiempo de crisis. Así, algunos estudios han desembocado en la representación cartográfica de los lugares percibidos como peligrosos por la población, dejando entrever distorsiones con los mapas producidos por los científicos (D’Ercole \& Rançon, 1994; D’Ercole \& Metzger, 2001; Salazar, 2008). Las condiciones de involucramiento de la sociedad civil y de los actores locales en la gestión de las alertas y de la comunicación de crisis en situación de incertidumbre también han sido analizadas (Metzger et al., 1999; Affeltranger \& Meschinet de Richemond, 2003). Por último, otros geógrafos han participado en la implementación de modelos de simulación a partir de SIG (Sistema de Información Geográfica). La aplicación puede ser útil en el campo de los seguros (Pico, 2006), para reducir la vulnerabilidad urbana, pero también para establecer planes de crisis (estimación de balances humanos y de los lugares afectados, estimación de la congestión de las vías, gestión del envío de los auxilios, etc.). De manera general, la geomática (teledetección, SIG, modelización geográfica) ha demostrado su utilidad para la prevención de los riesgos y algunas aplicaciones son directamente útiles para la gestión de las crisis (Montoya, 2003; D’Ercole et al., 2005; Demoraes et al., 2006; Gadal, 2006).

La investigación geográfica que ha abordado la crisis y su manejo tiene su relevancia pero no constituye generalmente el enfoque principal de la investigación. No ha sido emprendida ninguna geografía de los espacios y territorios de la gestión de las crisis y no existe evaluación alguna de la vulnerabilidad de los sistemas de gestión de crisis a partir del enfoque espacial. Los desafíos de tal investigación radican en reforzar los conocimientos científicos en este campo y en aportar nuevas luces sobre la complejidad e incertidumbre de las situaciones de crisis. 
Considerando las limitaciones de la prevención en cualquier parte del mundo, el tema del manejo de las crisis se torna progresivamente central y no solo en las ciudades de los países en desarrollo.

\section{LOS ESPACIOS DEL MANEJO DE CRISIS}

Puesto que la crisis dibuja espacios y flujos que ya no son los del funcionamiento normal, la investigación debe abordar frontalmente estos interrogantes: ¿Cuáles son los espacios del manejo de la crisis? ¿Cómo identificarlos? ¿Cómo se articulan estos espacios? Estas preguntas son más complejas de lo que parecen a primera vista, sobre todo cuando se trata de responder a ellas en la perspectiva de una crisis por venir y no durante o después. Los espacios de la crisis no son solamente aquellos que son susceptibles de ser afectados por un fenómeno destructor. En efecto, se ha de plantear la hipótesis que por lo menos dos tipos de espacios están construidos por el manejo de las crisis: los que se deben auxiliar en prioridad, es decir los que son más vulnerables; y los espacios recursos, aquellos que disponen de medios materiales y de decisión para protección y auxilio7.

\section{1. Los espacios por auxiliar}

Para definir los espacios que se deben auxiliar en prioridad con la perspectiva de una situación potencial de crisis, se plantea la hipótesis que los espacios a tomar en cuenta son los más vulnerables, es decir, los más susceptibles de ser afectados, y que presentan fragilidades identificables a priori y una población vulnerable. Esta vulnerabilidad de la población es definida en función de sus características propias, de condiciones espaciales particulares (accesibilidad), de la ocupación de territorios en los que el manejo de crisis es problemático y del grado de exposición a las amenazas. Los espacios vulnerables se agrupan en 4 clases:

- Los espacios en los que la población presenta vulnerabilidades en el plano demográfico (altas concentraciones humanas, población anciana, muy joven, discapacitada, etc.) y socioeconómico (pobreza, exclusión, acceso limitado a la información, etc.), en otros términos, en el plano de sus características propias (vulnerabilidad intrínseca). Los censos y las encuestas proveen los principales datos que permiten delimitarlos.

- Los espacios de difícil acceso, lo que puede impedir el ingreso o la salida de las zonas susceptibles de ser afectadas, y, en algunos casos, que pueden terminar totalmente aislados cuando se cortan las vías de acceso. La identificación de

7 Un tercer tipo podría ser mencionado: los espacios susceptibles de amplificar la crisis. Son aquellos que soportan los elementos esenciales del funcionamiento y del desarrollo urbano cuya pérdida o mal dinamismo podrían afectar, por efecto dominó, la organización urbana y amplificar la crisis, en gravedad y en duración (por ejemplo: aeropuertos, mercados mayoristas, lugares de almacenaje de hidrocarburos, lugares de producción mayor de la economía, etc.). Solo se cita este tipo de espacio para recordarlo, pues la reflexión todavía ha avanzado poco sobre este tema. 
estos espacios es el resultado del análisis de la red vial y de las condiciones de circulación, de la topografía, de la hidrografía y de los obstáculos antrópicos.

- Los espacios en los que son previsibles dificultades de manejo de crisis en el plano político e institucional. El manejo de crisis puede, por ejemplo, resultar problemático en el caso de conflictos territoriales, de la existencia de discontinuidades político administrativas que hacen difícil, incluso imposible, la intervención de las autoridades. La ocupación ilegal de tierras puede conducir a este tipo de dificultades. La determinación de estos espacios supone indagaciones y una profunda reflexión sobre los actores de la crisis y de su manejo y sobre las condiciones de implementación de las operaciones de socorro.

- Los espacios en los cuales las amenazas (de origen natural o antrópico, solas o en interacción) podrían manifestarse y, más particularmente, aquellas cuya probabilidad de ocurrencia y/o gravedad son más altas. Los datos provienen de diversos institutos científicos y técnicos que producen mapas de amenazas. Estos mapas pueden ser agrupados para desembocar en una cartografía multiamenazas que muestre cuántas de esta podrían afectar un espacio dado y que sugiera las posibilidades de interacción entre amenazas.

Una síntesis de estos cuatro espacios, que agrupa las cuatro formas de vulnerabilidad, evidenciará un grado de vulnerabilidad, y por consiguiente designará los que se encuentren en mayor riesgo: lugares con una población numerosa, poco accesible, muy expuestos a amenazas y donde se puedan detectar a priori dificultades de gestión de crisis.

\section{2. Los «espacios recursos» del manejo de crisis}

Los espacios recursos son definidos como los espacios donde se sitúan los elementos útiles, incluso indispensables, para el manejo de las crisis: centros de decisión, centros operacionales, recursos en atención medica de emergencia, abastecimiento de agua potable, de alimentos, vías de comunicación, telecomunicaciones, abastecimiento en energía, albergues, lugares de almacenamiento de la ayuda de emergencia, etc. Algunos recursos son fundamentales tanto en periodo de crisis como en periodo de funcionamiento normal de la ciudad: el abastecimiento de agua, las telecomunicaciones o los centros de salud. Otros son específicos y adquieren su relevancia en periodo de crisis: las instituciones de coordinación y de manejo, los organismos de operaciones de socorro, las fuentes alternativas de abastecimiento de agua, los albergues o los lugares de depósito de los escombros producidos por un desastre.

Los datos que permiten identificar y cartografiar los recursos de la crisis pueden existir de manera formal y localizada, por ejemplo en los planes de manejo de crisis, o en ficheros oficiales como los que están disponibles en ciertos ministerios - por ejemplo el ministerio de Salud que dispone de la información para localizar y caracterizar a los centros de salud de una ciudad-. Otros datos deben ser construidos a partir de un trabajo de campo, entrevistas y encuestas. Es importante 
«extraer» el conocimiento de los actores gestores de la crisis, en particular aquellos que son susceptibles de provocar discusiones porque están mal o no están formalizados (situación frecuente en relación a las zonas de albergue, por ejemplo). Los análisis de experiencias anteriores constituyen igualmente una base de información esencial.

Definir elementos y espacios recursos de la crisis supone plantear el tema de la escala geográfica en la que uno se coloca (escala metropolitana o de barrios). Lo que se considera esencial a escala de la aglomeración puede resultar secundario localmente y viceversa. Por ejemplo, una casa comunal puede constituir un recurso de crisis esencial como albergue o centro de decisión a escala de un barrio, y al mismo tiempo no revestir ninguna importancia particular a escala de una aglomeración. En consecuencia, los elementos efectivamente útiles para el manejo de crisis son a priori múltiples y están diseminados sobre el territorio. Este tema es importante porque más allá de la identificación y de la localización de todos los recursos movilizables para enfrentar una situación de emergencia, se plantea la cuestión de los elementos esenciales del manejo de crisis a escala de un territorio urbano.

Es indispensable la identificación de los elementos esenciales del manejo de crisis a escala de un territorio para identificar, delimitar, preparar y proteger los espacios estratégicos para el manejo de las crisis a escala de un conjunto urbano, es decir los lugares que concentran elementos esenciales de crisis. Estos últimos pueden ser determinados con los actores institucionales del manejo de crisis utilizando criterios cuantitativos (por ejemplo, centros de salud en función del número de camas), cualitativos (elementos importantes en el plano funcional: por ejemplo, centros de salud que disponen de servicios particularmente útiles en periodo de crisis, como una unidad de quemados) o territoriales (por ejemplo, centros de salud poco importantes, cuantitativa o cualitativamente, pero que prestan servicios a un territorio extenso).

\section{3. La articulación entre espacios vulnerables y espacios recursos}

El manejo de crisis consiste, muy concretamente, en relacionar estos dos tipos de espacios que agrupan por un lado las necesidades de auxilio (los espacios vulnerables) y, por el otro, los recursos que permiten responder a estas necesidades en situación de emergencia (los espacios recursos). El análisis de sus articulaciones espaciales supone la constitución de una base de datos georeferenciados y el uso de un sistema de información geográfica (SIG). Permite evidenciar, por un lado, las vulnerabilidades del manejo de crisis ligadas a los problemas que surgen de esta relación. En otras palabras, ¿en qué medida, los espacios vulnerables tienen acceso a los recursos? ¿Cómo llegan los recursos a los espacios que los necesitan? Hay que reflexionar al mismo tiempo en términos de dependencia y de autonomía de los espacios vulnerables en relación con los recursos, pues pueden disponer localmente de recursos de manejo de crisis. 
Por otro lado permite identificar los espacios críticos del manejo de crisis, es decir, susceptibles de estar en gran dificultad en periodo de crisis, no solo por el hecho de su propia vulnerabilidad (sociodemográfica, institucional, accesibilidad, etc.) sino también por sus dificultades de acceso a los recursos del manejo de crisis, identificables a priori.

La vulnerabilidad de los recursos del manejo de crisis debe igualmente ser considerada porque contribuye a acrecentar el carácter crítico de los espacios que dependen de ellos. Su análisis permite identificar espacios generadores de vulnerabilidades en periodo de crisis, donde se sitúan elementos esenciales de manejo de crisis que habrán sido identificados como vulnerables. En razón de su importancia para enfrentar una situación de crisis y en razón de su vulnerabilidad, estos elementos y espacios, en caso de daños, van a afectar todo el manejo de la crisis y transmitir su vulnerabilidad a gran parte del territorio urbano, incluso a su conjunto.

\section{DIMENSIONES ESPACIALES DEL MANEJO DE CRISIS: APLICACIÓN EN QUITO}

La reflexión sobre la dimensión espacial del manejo de las crisis recién comienza y las vulnerabilidades de los sistemas de manejo no son abordados desde el ángulo espacial y territorial sino de manera marginal. Proponemos dos ejemplos de aplicación dentro del marco del programa «Sistema de Información y Riesgos en el Distrito Metropolitano de Quito $(\mathrm{DMQ}) »$, realizado en colaboración entre el IRD y la Municipalidad de Quito (1999-2004)8. Estas reflexiones prosiguen en el marco del programa PACIVUR en Quito, Lima y La Paz.

\section{1. Espacios del manejo de crisis en Quito}

En Quito, los elementos útiles para el periodo de crisis han sido agrupados en seis grandes categorías:

- Los centros y organismos de decisión, de coordinación y de intervención en situación de crisis.

- El abastecimiento alimenticio y de agua.

- Los elementos de apoyo a la población (centros de salud y albergues).

- El abastecimiento energético (combustibles, electricidad).

- Las comunicaciones (movilidad, telecomunicaciones).

- Los elementos útiles para el periodo de recuperación (empresas que disponen de máquinas para remoción de escombros, que comercializan materiales de construcción, canteras).

8 Esta investigación se centró principalmente en los elementos esenciales del funcionamiento y del desarrollo de Quito y su vulnerabilidad en una perspectiva de planificación preventiva urbana. Sin embargo, el tema de la crisis ha sido considerado de manera exploratoria. 
La investigación ha permitido la constitución de una base de datos georeferenciados que corresponden a estos elementos y la realización de un gran número de mapas analíticos y sintéticos (D’Ercole \& Metzger, 2004). Estos atañen al conjunto de los elementos útiles pero también a los que han sido identificados como elementos esenciales («indispensables», «estratégicos») del manejo de crisis a escala del distrito metropolitano (cuadro 1).

Cuadro 1 - Criterios de identificación de elementos esenciales de manejo de crisis en el DMQ

\begin{tabular}{|c|c|}
\hline $\begin{array}{l}\text { Elementos esenciales } \\
\text { de manejo de crisis }\end{array}$ & Elementos esenciales considerados \\
\hline $\begin{array}{l}\text { Centros de decisión } \\
\text { e intervención }\end{array}$ & Todos los centros de decisión e intervención identificados \\
\hline \multirow[b]{2}{*}{$\begin{array}{l}\text { Abastecimiento de } \\
\text { alimentos y de agua }\end{array}$} & $\begin{array}{l}\text { Los elementos esenciales del funcionamiento habitual del distrito para } \\
\text { el abastecimiento alimenticio así como todos los lugares inventariados } \\
\text { de almacenamiento de víveres provenientes de la ayuda nacional e } \\
\text { internacional }\end{array}$ \\
\hline & $\begin{array}{l}\text { Los elementos esenciales del funcionamiento habitual del distrito } \\
\text { para el abastecimiento de agua, a los que se agregan: las plantas } \\
\text { Noroccidente y Conocoto, los tanques de volumen superior a } 1000 \mathrm{~m}^{3} \\
\text { (no considerados entre los elementos esenciales de funcionamiento), } \\
\text { los pozos cuyo caudal es superior a } 15 \mathrm{~m}^{3} / \mathrm{s} \text { así como el sitio La Ofelia } \\
\text { que permite cargar tanqueros. }\end{array}$ \\
\hline \multirow{2}{*}{$\begin{array}{l}\text { Apoyo a la población } \\
\text { (salud, albergues) }\end{array}$} & $\begin{array}{l}\text { Los establecimientos de salud considerados esenciales para el } \\
\text { funcionamiento del DMQ, así como los lugares de gestión de } \\
\text { ambulancias. }\end{array}$ \\
\hline & $\begin{array}{l}\text { Los albergues (permanentes y temporales) considerados como los más } \\
\text { importantes en el estudio IRD, Dirección Metropolitana de Salud y } \\
\text { Junta Provincial de Defensa Civil (2003). }\end{array}$ \\
\hline \multirow[b]{2}{*}{$\begin{array}{l}\text { Abastecimiento } \\
\text { energético } \\
\text { (electricidad, } \\
\text { combustibles) }\end{array}$} & $\begin{array}{l}\text { Los elementos esenciales de funcionamiento del DMQ en materia de } \\
\text { suministro de energía eléctrica }\end{array}$ \\
\hline & $\begin{array}{l}\text { Los elementos esenciales de funcionamiento del DMQ en materia de } \\
\text { abastecimiento de combustibles, completados con las gasolineras de } \\
5 \text { a } 9 \text { surtidores, las estaciones centralizadas y lugares de expendio de } \\
\text { gas de una capacidad de almacenamiento superior a } 10 \mathrm{~m}^{3} \text {, la reserva } \\
\text { de combustible del aeropuerto }\end{array}$ \\
\hline \multirow{2}{*}{$\begin{array}{l}\text { Comunicaciones } \\
\text { (movilidad, } \\
\text { telecomunicaciones) }\end{array}$} & $\begin{array}{l}\text { El aeropuerto, los ejes viales esenciales del funcionamiento del DMQ } \\
\text { así como los ejes de segundo nivel }\end{array}$ \\
\hline & $\begin{array}{l}\text { Los elementos esenciales de funcionamiento del DMQ en materia de } \\
\text { telecomunicaciones }\end{array}$ \\
\hline \multirow{3}{*}{$\begin{array}{l}\text { Elementos útiles } \\
\text { para el período de } \\
\text { recuperación }\end{array}$} & $\begin{array}{l}\text { Las empresas que disponen de al menos } 20 \text { unidades de maquinaria } \\
\text { útil para la limpieza, la demolición y la construcción }\end{array}$ \\
\hline & $\begin{array}{l}\text { Los lugares de mayor concentración de las empresas comercializadoras } \\
\text { de materiales útiles para la construcción }\end{array}$ \\
\hline & Las canteras de más de 10 ha \\
\hline
\end{tabular}


Entre los mapas sintéticos, el mapa de los lugares esenciales del manejo de las crisis (fig. 1) señala una fuerte concentración de elementos de manejo definidos en el cuadro 1. Estos últimos ocupan $7 \%$ del espacio metropolitano y se concentran en $2 \%$ de este espacio9. El mapa pone de relieve el centro (desde el centro histórico

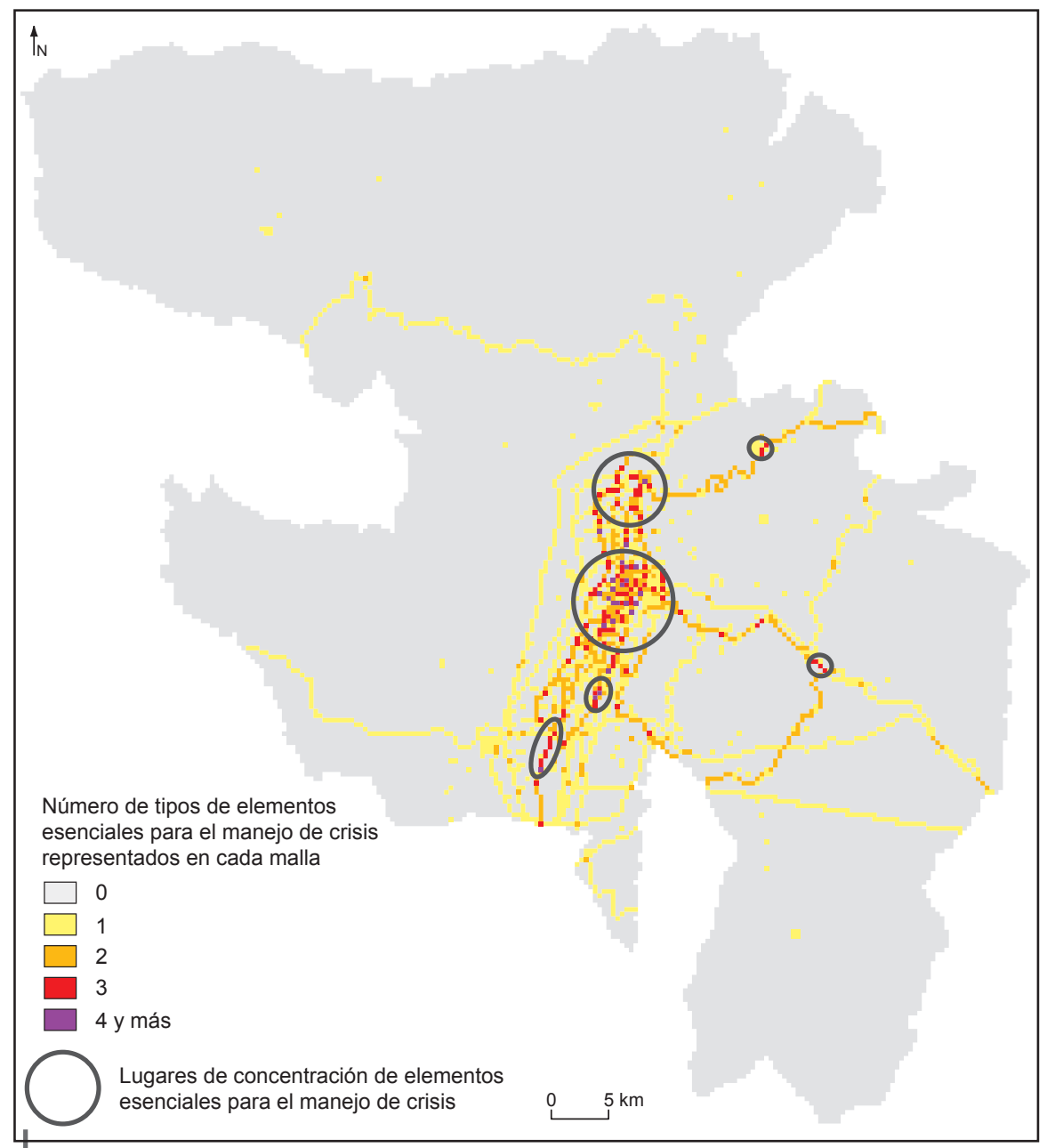

Figura 1 - Lugares esenciales para el manejo de crisis en el DMQ

9 El mapa ha sido construido a partir del recorte del espacio metropolitano en 28887 mallas cuadradas de $400 \mathrm{~m}$ de lado. Para cada uno de los seis campos considerados (cuadro 1), las mallas han sido caracterizadas por una variable binaria que indica la presencia (valor 1) o la ausencia (valor 0) del tipo de elemento esencial considerado. Así, una malla puede soportar, en teoría, de 0 a 6 tipos de elementos esenciales. Estos elementos ocupan 2051 mallas, es decir, 7,1 \% del espacio metropolitano (para mayores detalles sobre la metodología, ver D`Ercole \& Metzger, 2004). 
hasta el aeropuerto) y el norte de la ciudad de Quito así como espacios más reducidos y más lineales en el sur y los valles orientales. El mapa siguiente (fig. 2) establece una relación espacial entre los elementos esenciales del funcionamiento urbano normal y los elementos esenciales del manejo de crisis, lo que permite evidenciar espacios especializados.

El espacio central, que va desde el centro histórico hasta el aeropuerto, está marcado por la presencia de elementos esenciales de funcionamiento normal aún cuando los elementos de crisis son numerosos. Estos últimos están generalmente ubicados en las mismas mallas y desempeñan un rol determinante, en particular, en los campos de la decisión o de la atención médica. Los sectores periféricos (el norte de la ciudad pero también algunos sectores meridionales) son espacios más específicos del manejo de las crisis. Fuera de la ciudad de Quito, los espacios en los que se concentran elementos esenciales, sea cual fuere su tipo, son poco numerosos, pero los elementos esenciales de crisis son más visibles.

El norte de Quito, más allá del aeropuerto, particularmente a nivel del intercambiador de Carcelén y de sus prolongaciones viales, se muestra como un espacio especializado en elementos esenciales de manejo de crisis — detallados en la figura 3-.

El número y la variedad de los elementos de crisis son impresionantes. Se puede observar la importancia de las redes, sobre todo de las vías cuyo rol es decisivo en periodo de crisis, ya sea para el abastecimiento alimenticio, de combustibles, para mandar auxilios o para evacuar cuando es necesario. Pero también se encuentran numerosos elementos relacionados con el abastecimiento energético (líneas de transmisión eléctrica, almacenamiento de gas, estaciones de gasolina) y de agua potable (reservorios, el sector de La Ofelia adaptado para el abastecimiento de camiones cisterna), albergues, telecomunicaciones, sistema operativo (cuarteles de bomberos, administración zonal), etc., sin olvidar el aeropuerto, pieza vital en periodo de crisis.

Cabe resaltar la importancia de este espacio, aproximadamente $50 \mathrm{~km}^{2}$, y la necesidad de una atención particular por parte de las autoridades municipales en términos preventivos pues su no funcionamiento total o parcial constituiría un gran problema para el distrito metropolitano en caso de emergencia. De esto se desprende claramente la articulación entre la prevención de los riesgos y el manejo de las crisis.

\section{2. La articulación espacios vulnerables/espacios recursos médicos}

La eficiencia del manejo de una crisis depende en gran parte de la calidad de la articulación entre las necesidades de auxilio situadas en los espacios vulnerables y la oferta en recursos de manejo de crisis. 


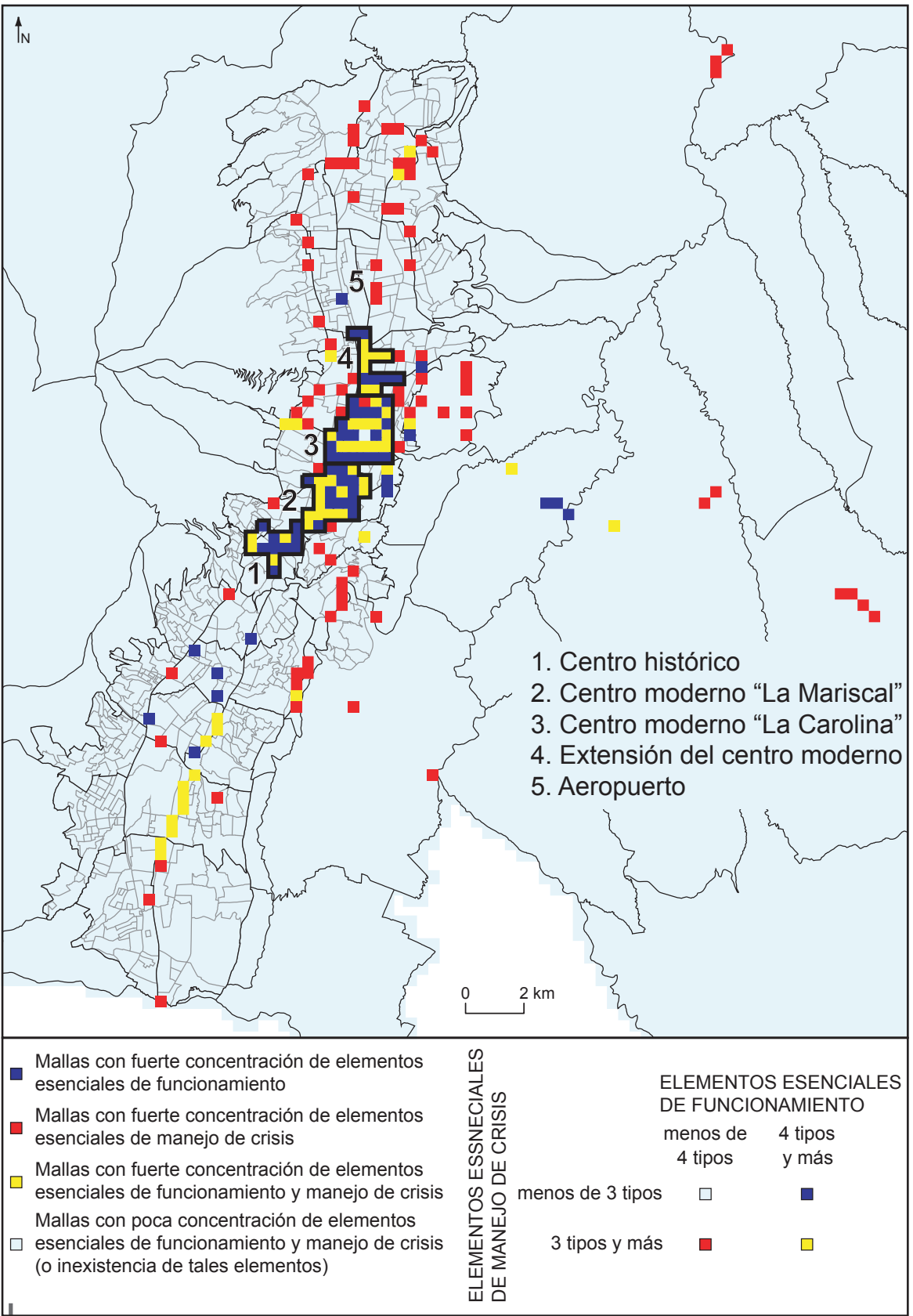

Figura 2 - Lugares de concentración de los elementos esenciales de funcionamiento y de manejo de crisis en el DMQ 


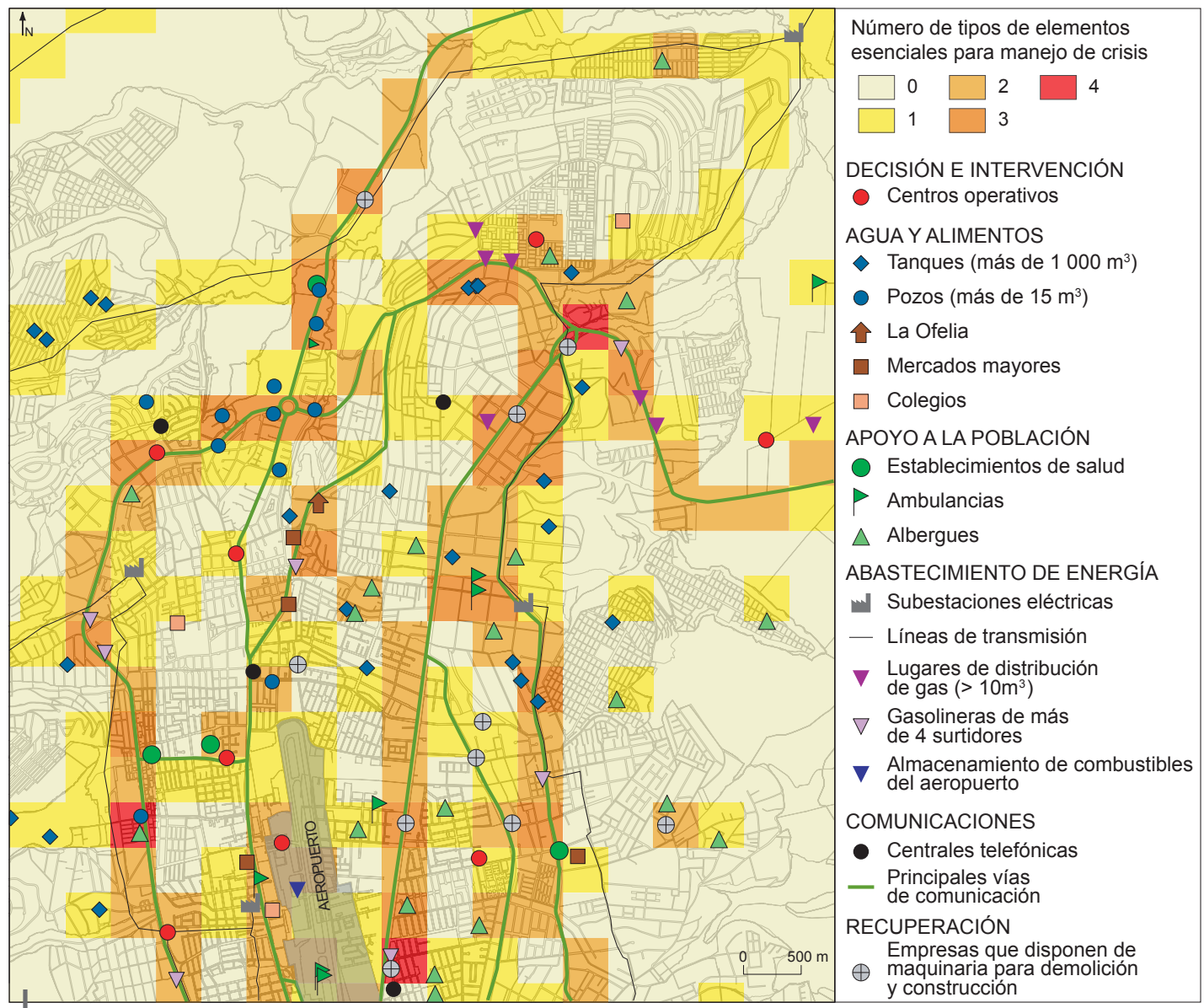

Figura 3 - Los elementos esenciales para el manejo de crisis en el norte de Quito

Respecto a los recursos médicos en Quito, por ejemplo, esta articulación puede ser observada en el territorio (fig.s 4A, B y C). La figura 4A que establece una relación entre la densidad de población y los centros de salud, muestra que el Distrito Metropolitano de Quito dispone de una amplia cobertura de servicios de salud a pesar de algunos vacíos en el norte y en el sur de la ciudad así como en las parroquias suburbanas. En realidad, la situación es más crítica de lo que parece a primera vista, en la medida en que esta constatación se basa en todos los establecimientos de salud desde los centros de atención más elementales hasta los mayores hospitales. En efecto, numerosos establecimientos no disponen de camas ni de recursos suficientes para afrontar una situación de emergencia, sea cual fuere su magnitud. La figura $4 \mathrm{~B}$, que se basa únicamente en los centros de salud que disponen de camas, refleja mejor las dificultades que puede conocer el distrito en periodo de crisis. Solamente una parte de la ciudad de Quito (en particular, el centro y el centro norte) puede contar con centros de salud de 


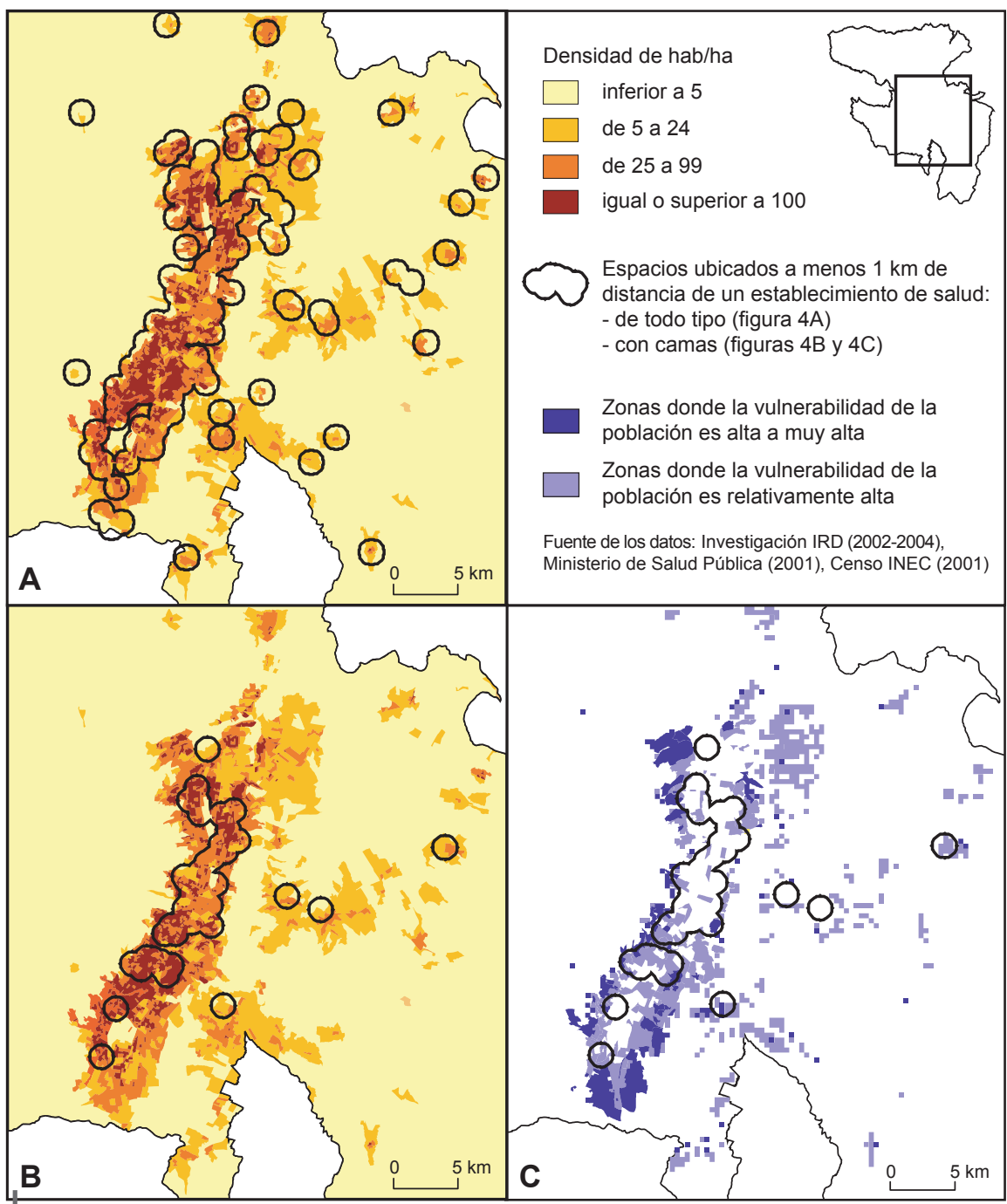

Figura 4A - Densidad de población y cobertura hospitalaria en el DMQ (centros de salud de todo tipo)

Figura 4B - Densidad de población y cobertura hospitalaria en el DMQ (centros de salud con camas)

Figura 4C - Vulnerabilidad de la población y cobertura hospitalaria en el DMQ (centros de salud con camas) 
buena calidad sin que sean necesarios largos desplazamientos. Peor aún es la articulación espacial entre los centros de salud y la población más vulnerable del distrito10 (fig. 4C). La casi totalidad de esta población se encuentra a más de $1 \mathrm{~km}$ de un centro de salud con capacidad hospitalaria, lo que constituye un caso muy claro de exclusión social que agrava la vulnerabilidad de poblaciones ya marginalizadas.

\section{CONCLUSIÓN}

Este artículo es una primera tentativa para destacar el interés del enfoque espacial del manejo de crisis y la necesidad de desarrollar investigaciones sobre este tema. El análisis de la articulación espacial entre espacios vulnerables y espacios recursos resulta muy útil para evidenciar las vulnerabilidades del sistema de manejo de crisis. Este permite reflexionar en términos de preparación para el manejo de crisis y de ayuda a la decisión estableciendo, al mismo tiempo, puentes con la prevención de riesgos. En el caso de los centros de salud de Quito, se puede tratar de reforzar los centros menores de atención médica cuya cobertura espacial es demasiado extensa en relación con las posibilidades de intervención. También se puede tratar de prever la implantación de hospitales móviles, en lugares escogidos por no tener estableciemientos de salud y por ser densamente poblados. En consecuencia, el enfoque espacial del manejo de crisis permite reflexionar simultáneamente en términos de planificación preventiva urbana y de preparación para las crisis (por ejemplo, optimizar la distribución territorial de los centros de salud en función de las necesidades, hacer que las porciones del territorio, susceptibles de estar aisladas en periodo de crisis, sean autónomas en servicios básicos, etc.). Esto demuestra que existe una articulación muy clara entre actividades que a menudo se consideran de manera separada: el manejo de crisis y su preparación de un lado, la prevención y la planificación preventiva territorial del otro. Este enfoque plantea igualmente el tema de las escalas de manejo de crisis y permite diferenciar los tipos de recursos entre los que están localmente necesarios, es decir los que deben ubicarse lo más cerca de los espacios vulnerables, y los que suponen una localización y una organización para responder a las necesidades del territorio urbano en su conjunto.

10 Esta vulnerabilidad toma en cuenta criterios sociodemográficos, criterios de capacidad de manejo de crisis, accesibilidad de la población y exposición a las amenazas de origen natural y antrópico (D’Ercole \& Metzger, 2004). 


\section{Referencias citadas}

AFFELTRANGER, B. \& MESCHINET, N., 2003 - Gestion de l'alerte et de l'information lors des crues : conditions et limites d'une démarche participative locale. L'exemple des Deux-Sèvres. Flux, 51: 16-27.

ALEXANDER, D., 2000 - Confronting catastrophe: new perspectives on natural disasters, 288 pp.; Oxford: Oxford University Press.

APPERT, M. \& CHAPELON, L., 2008 - La vulnérabilité des réseaux routiers urbains face au risque d'altération. Géorisques, 2; Montpellier: Presses Universitaires de la Méditerranée.

BIMAL KANTI, P. , 2005 - Evidence against disaster-induced migration: the 2004 tornado in north-central Bangladesh. Disasters, 29 (4): 370-385; Oxford: Blackwell.

BLAIKIE, P., CANNON, T., DAVIS, I. \& WISNER, B., 1994 - At risk. Natural people's vulnerability and disasters, 280 pp.; Nueva York: Routledge.

CHARDON, A.-C., 1996 - Croissance urbaine et risques «naturels». Évaluation de la vulnérabilité à Manizales, Andes de Colombie, 387 pp.; Grenoble: Univ. J. Fourier. Thèse de doctorat.

CURTIS, A., MILLS, J. W., KENNEDY, B., FOTHERINGHAM, S. \& MCCARTHY, T., 2007 Understanding the Geography of Post-Traumatic Stress: An Academic Justification for Using a Spatial Video Acquisition System in the Response to Hurricane Katrina. Journal of Contingencies and Crisis Management, 15 (4): 208-219.

CUTTER, S-L., MITCHELL, J. T. \& SCOTT, M. S., 2000 - Revealing the vulnerability of people and place: a case study of Georgetown County, South Carolina. Annals of the Association of American Geographers, 90 (4): 713-737.

DEMORAES, F., 2004 - Mobilité, enjeux et risques dans le District Métropolitain de Quito (Équateur), 587 pp.; Chambéry: Université de Savoie. Thèse de doctorat en Géographie.

DEMORAES, F., D’ERCOLE, R., METZGER, P. \& SOURIS, M., 2006 - Enjeux, mobilité, accessibilité et risques. L'exemple du District Métropolitain de Quito (Équateur). Revue Internationale de Géomatique, 16 (3-4): 435-456.

DENIS, H., 1993 - Gérer les catastrophes. L'incertitude à apprivoiser, 248 pp.; Montreal: Les Presses de l'Université de Montréal. Collection Intervenir.

D'ERCOLE, R., 1991 - Vulnérabilité des populations face au risque volcanique. Le cas de la région du volcan Cotopaxi (Équateur), 460 pp.; Grenoble: Université Joseph Fourier. Thèse de doctorat.

D'ERCOLE, R., 1994 - Les vulnérabilités des sociétés et des espaces urbanisés : concepts, typologie, modes d'analyse. Revue de Géographie Alpine, 4 (LXXXII): 87-96.

D'ERCOLE, R., 1996 - La catastrophe et son impact a moyen terme : I'exemple de Popayan (Colombie), dix ans après le séisme du 31 mars 1983». Cahiers des Sciences Humaines, 96 (2): 445-470; Orstom.

D'ERCOLE, R. \& METZGER, P., 2000 - La vulnérabilité de Quito (Équateur) face à l'activité du Guagua Pichincha. Les premières leçons d'une crise volcanique durable. Cahiers Savoisiens de Géographie, 1: 39-52; Université de Savoie.

D’ERCOLE, R. \& METZGER, P. , 2001 - Différenciations spatiales et sociales, représentations et gestion du risque volcanique à Quito. Actes du séminaire international Risques et Territoires, 3: 237-250.

D'ERCOLE, R. \& METZGER, P., 2004 - Vulnerabilidad del Distrito Metropolitano de Quito, 496 pp.; Quito: MDMQ, IRD. Colección Quito Metropolitano. 
D’ERCOLE, R. \& METZGER, P., 2009 - La vulnérabilité territoriale : une nouvelle approche des risques en milieu urbain. Cybergeo. Vulnérabilités urbaines au sud <http://www. cybergeo.eu/index22022.html>.

D'ERCOLE, R. \& MONCAYO, J. F., 1991 - «Influents locaux» face à une situation d'urgence : une analyse selon I'hypothèse d'une éruption du volcan Cotopaxi (Équateur). Bulletin de I'Institut Français d'Études Andines, 20 (1): 181-220.

D’ERCOLE, R. \& RANÇON, J.-P., 1994 - La future éruption de la montagne Pelée: Risques et représentations. Mappemonde, 4: 31-36.

D`ERCOLE, R., CHANDES, J., PERFETTINI, H. \& AUDIN, L., 2007 - Le séisme de Pisco du 15 août 2007. Entre urgence et reconstruction. EchoGéo, $3<$ http://prodig.univparis1.fr/publications_umr/EchoGeo/numero3/Articles/Dercole.pdf $>$.

D’ERCOLE, R., METZGER, P. \& BERMúDEZ ARBOLEDA, N., 2005 - Espaces enjeux, espaces vulnérables. Le cas de Quito (Équateur). In: ISTED. Systèmes d'information géographique et gestion des risques: 16

DIRECCIÓN GENERAL DE PROTECCIÓN CIVIL, 1997 - Normativa Básica Española sobre Protección Civil, 190 pp.; España: Protección Civil.

DUBOIS-MAURY, J. \& CHALINE, C., 2002 - Les risques urbains, 208 pp.; París: Armand Colin.

GADAL, S., 2006 - Pertinence de la géomatique pour la prévention et la gestion des risques naturels. Actes du Colloque Géographes et assureurs face aux risques naturels: 192-221; Niort: Fondation MAIF.

GAILLARD, J.-C., 2001 - Implications territoriales et socio-économique des crises et des réponses liées à l'éruption et aux lahars du Mont Pinatubo, 1991-2001 (Philippines); Chambéry: Université de Savoie. Thèse de doctorat.

GAILLARD, J.-C., LIAMZON, C. C. \& VILLANUEVA, J. D., 2007 - Natural disaster? A retrospect into the causes of the late-2004 typhoon disaster in Eastern Luzon, Philippines. Environmental Hazards, 7 (1): 1-14.

GILBERT, C., 2005 - L'analyse des crises : entre normalisation et évitement. In: Risques, crises et incertitudes : pour une analyse critique (O. Borraz, C. Gilbert \& P-B. Joly, eds.): 175-223; Cahiers du GIS Risques Collectifs et Situations de Crise.

GODARD, O., HENRY, C., LAGADEC, P. \& MICHEL-KERJAN, E., 2002 - Traité des nouveaux risques, 620 pp.; París: Ed. Gallimard.

GRIOT, C., 2007 - Vulnérabilité et transport de matières dangeureuses : une méthode d'aide à la décision issue de l'expertise de la Sécurité Civile. CyberGéo : Revue Européenne de Céographie, n. ${ }^{\circ}$ 361. http://www.cybergeo.eu/docannexe/file/5995/361.pdf

KERVEN,G. Y. \& WYBO, J.-L., 2002 - Le retour d'expérience de la gestion des crises : d'octobre 1986 à septembre 2001 <http://mines-energie.org/Dossiers/ Nucl2003_18.pdf >.

LAGADEC, P., 2003 - La recherche confrontée à la question des crises. In: Risques collectifs et situations de crise. Apports de la recherche en sciences humaines et sociales (Claude Gilbert, ed.): 297-316; París: L'Harmattan. Collections Risques collectifs et situations de crise.

LAGADEC, P., 2007 - Katrina : Examen des rapports d'enquête. Tome 1: A Failure of Initiative, 140 pp.; École Polytechnique. Cahiers du Laboratoire d'Économétrie.

LANE, L. R., TOBIN G. A. \& WHITEFORD, L. M., 2003 - Volcanic hazard or economic destitution: hard choices in Baños, Ecuador. Environmental Hazards, 5: 23-34.

LAVIGNE, F., PARIS, R. (eds.), 2006 - Le tsunami du 26 décembre 2004 en Indonésie. Rapport scientifique du programme Tsunarisque (2005-2006), 306 pp.; Délégation Interministérielle pour I’aide Post-Tsunami (DIPT), Ambassade de France en Indonésie. 
LUTOFF, C., 2000 - Le système urbain niçois face à un séisme : méthode d'analyse des enjeux et des dysfonctionnements potentiels, 368 pp.; Chambéry: Université de Savoie. Thèse de doctorat.

MANCEBO, F., 2006 - Katrina et la Nouvelle-Orléans : entre risque «naturel» et aménagement par l'absurde. Cybergeo, art. 353 < http://www.cybergeo.eu/index90.html >.

METZGER, P., D'ERCOLE, R. \& SIERRA, A., 1999 - Political and scientific uncertainties in volcanic risk management: The yellow alert in Quito in October 1998. Geojournal, 49: 213-221.

MEUR-FEREC, C., DEBOUDT, P., DELDREVE, V., FLANQUART, H., HELLEQUIN, A.-P., HERBERT, V., LONGUEPEE, J. \& MOREL, V., 2004 - La vulnérabilité des territoires côtiers : évaluation, enjeux et politiques publiques, 56 pp.; Programme National Environnement Côtier. Rapport final.

MONTOYA, L., 2003 - Geodata acquisition through mobile GIS and digital video: an urban disaster management perspective. Environmental Modelling \& Software, 18: 869-876.

MOREL, V., DEBOUDT, P., HELLEQUIN, A.-P., HERBERT, V. \& MEUR-FEREC, C., 2006 Regard rétrospectif sur l'étude des risques en géographie à partir des publications universitaires (1980-2004). L'Information Géographique, 70: 6-24.

O'HARE, G. \& RIVAS, S., 2005 - The landslide hazard and human vulnerability in La Paz City, Bolivia. The geographical Journal, 171 (3): 239-258.

OZIER, P. \& DE LONGUEVILLE, F., 2005 - Tsunami en Asie du Sud-Est : retour sur la gestion d'un cataclysme naturel apocalyptique. Cybergeo, art. $321<$ http://www.cybergeo. eu/index3081.html $>$.

PELLING, M., 2003 - The vulnerability of cities: natural disasters and social resilience, 256 pp.; Londres: Earthscan.

PICO, L., 2006 - Gestion assurantielle et aménagement urbain : I’usage des SIG appliqués au risque sismique à Beyrouth collectifs. Actes du Colloque Géographes et assureurs face aux risques naturels: 176-190; Niort: Fondation MAIF.

PIGEON, P., 1996 - La gestion des risques urbains. In: Risques naturels, risques de sociétés (A. Bailly, ed.): 51-62; París: Economica.

PIGEON, P., 2006 - L'efficacité des politiques gérant les risques dits naturels en France : lecture géographique. In: La vulnérabilité des sociétés et des territoires face aux menaces naturelles (Leone \& Vinet, eds.): 27-34; Montpellier. Col. Géorisques n 1.

POTTIER, N., 2006 - L'enquête et le diagnostic de vulnérabilité, outils de connaissance des risques et de prévention aux échelons individuels et collectifs. Actes du Colloque Géographes et assureurs face aux risques naturels: 134-165; Niort: Fondation MAIF.

PROVITOLO, D., 2005 - Un exemple d'effets de dominos : la panique dans les catastrophes urbaines. Cybergeo, art. 328 <http://www.cybergeo.eu/index2991.html>.

QUARANTELLI, E. L. (ed.), 1998 - What is a disaster? Perspectives on the question, 312 pp.; Nueva York: Routledge.

ROCHA, J. L. \& CHRISTOPLOS, I., 2001 - Disaster mitigation and preparedness on the Nicaraguan post-Mitch agenda. Disasters, 25 (3): 240-250.

SALAZAR, D., 2008 - Percepción de amenazas y riesgos de la población del Valle de los Chillos (Quito, Ecuador) expuesta al volcán Cotopaxi, 94 pp.; Quito: IFEA, PUCE. Mémoire de diplôme d'ingénieur géographe.

SAURI-PUJOL, D., ROSET-PAGES, D., RIBAS-PALOM, A. \& PUJOL-CAUSSA, P., 2001 - The escalator effect in flood policy: the case of the Costa-Brava. Applied geography, 21: 127-143; Catalonia. 
Las dimensiones espaciales del manejo de crisis. Interés de la investigación y aplicación en Quito

SERRANO, T., 2007 - La gestion du risque lié aux lahars du volcan Cotopaxi dans la vallée de Los Chillos (Équateur). Mise en évidence de facteurs institutionnels de vulnérabilité, 105 pp.; Montpellier: Université Paul Valéry. Mémoire de Master II, Gestion des catastrophes et risques naturels.

SIERRA, A., 2000 - Gestion et enjeux des espaces urbains à risque d'origine naturelle : les versants et les «quebradas» de Quito, Équateur, 326 pp.; Université de Paris 8. Thèse de doctorat

TUNSTALL, S., 2004 - La gestion des inondations en Angleterre et au Pays de Galles. In: Risques naturels et aménagement en Europe (Veyret et al., ed.): 88-107: París: A. Colin.

VEYRET, Y., GARRY, G., MESCHINET de RICHEMOND, N. (eds.), 2004 - Risques naturels et aménagement en Europe, 254 pp.; París: A. Colin.

VOIGHT, B., 1990 - The 1985 Nevado del Ruiz volcano catastrophe: anatomy and retrospection. Journal of Volcanology and Geothermal Research, 42: 51-188.

VOIRON-CANICIO, C. \& OLIVIER, F., 2005 - Anticiper, à l'aide d'un SIG, les conséquences de la paralysie urbaine en temps de catastrophe : application à la ville de Nice. In: Systèmes d'information géographique et gestion des risques: 55-58; París: Isted.

WALKER, B., HOLLING, C. S., CARPENTER, S. R. \& KINZIG, A., 2004 - Resilience, adaptability and transformability in social-ecological systems. Ecology and Society, 9 (2): 5 <http://www.ecologyandsociety.org/vol9/iss2/art5>.

WEICHSELGARTNER, J., 2001 - Disaster mitigation: the concept of vulnerability revisited Disaster Prevention and Management, 10 (2): 85-94.

WERRITTY, A., 2006 - Sustainable flood management: oxymoron or a new paradigm. Area, 38 (1): 16-23. 